\title{
Osteoscopic Surgery of Giant Cell Tumor of Bone for Preservation of Proximal Fibula
}

\author{
HIROYUKI FUTANI, SHUNSUKE KUMANISHI, GO MINAKAWA and SHINICHI YOSHIYA
}

Department of Orthopaedic Surgery, Hyogo College of Medicine, Nishinomiya, Japan

\begin{abstract}
Background: Osteoscopy is a minimally-invasive endoscopic technique for inspecting lesions inside bone marrow cavities. We describe the feasibility of osteoscopic surgery of giant cell tumor of bone (GCTB) in order to preserve the proximal fibula, and thereby achieve immediate recovery and avoid complications. Patients and Methods: Five patients with GCTB in the proximal fibula were treated using osteoscopic curettage with adjuvants (argon plasma coagulation and cementation). Functional outcome was evaluated by knee stability, Musculoskeletal Tumor Society (MSTS) rating, and Tegner score. Oncological outcome was evaluated for local recurrence and pulmonary metastasis. Results: Regarding functional outcome, knee instability was negative in all cases. Mean MSTS rating was 100\%. Tegner scores were the same as those prior to surgery. Neither local recurrence nor pulmonary metastasis were found. Conclusion: Osteoscopic surgery is feasible for immediate and complete recovery, and can improve quality of life for patients with GCTB of the proximal fibula with satisfactory oncological outcome.
\end{abstract}

Giant cell tumor of bone (GCTB) is an intermediate, locally aggressive but rarely metastasizing tumor, accounting for approximately $6 \%$ of all primary bone tumors. The proximal fibula is a relatively uncommon site of GCTB, accounting for only $2 \%$ of all GCTBs (1).

The options for surgical treatment of GCTB are en bloc resection and open curettage with or without adjuvants. When a GCTB develops at the proximal fibula, en bloc resection followed by reconstruction of the lateral collateral ligament (LCL) and the biceps tendon (BT) has been widely used to reduce the local recurrence rate (2-4). However, the two main concerns associated with this surgery are postoperative

Correspondence to: Dr. Hiroyuki Futani, Department of Orthopaedic Surgery, Hyogo College of Medicine, 1-1 Mukogawa Nishinomiya, Hyogo 663-8501, Japan. Tel: +81 798456452, Fax: +81 798456453, e-mail: h-futani@hyo-med.ac.jp

Key Words: Osteoscopy, endoscopy, giant cell tumor, fibula. peroneal nerve palsy and knee instability (4). In addition to these complications, a long period of rehabilitation, including immobilization for 2 to 6 weeks, is required due to reconstruction of the LCL and the BT $(4,5)$. Therefore, preservation of the proximal fibula is the ideal management for patients with GCTB with respect to knee stability, avoidance of peroneal nerve palsy and short rehabilitation period. All these are factors that can dramatically improve the quality of life for the patients.

Osteoscopy is a minimally-invasive endoscopic technique for inspecting lesions located inside the bone marrow cavity (6-10). In the current study, we applied osteoscopic sugery, that included combined curettage with adjuvants of argon plasma coagulation (APC) and cementation, in five patients with GCTBs of the proximal fibula. Our main purpose was to evaluate the feasibility of this technique for preservation of the proximal fibula in order to obtain immediate functional recovery and to reduce complications, in addition to improve oncological outcome.

\section{Patients and Methods}

Between 2000 and 2015, 59 patients with histologically proven GCTB were treated at our Institute. Five out of these GCTBs $(8.5 \%)$ had developed from the proximal fibula. They all underwent osteoscopic surgery, and had a complete follow-up period of minimum 2 years. The indication for osteoscopic surgery is a tumor located inside a bone cavity surrounded by a cortical wall, even if this is thin. The cortical wall provides safe protection of surrounding normal tissue which otherwise may be damaged by the surgery. However, one of our patients (case 5 in Table I) had anterior cortical destruction with extra-skeletal lesions. In such a case, denosumab can be used pre-operatively to create a sclerotic wall. Denosumab is a fully human monoclonal antibody with high affinity for receptor activator of nuclear factor kappa-B ligand, and therapeutic potential for treatment of GCTB has been pre-operatively reported (11). Our patient received denosumab $120 \mathrm{mg}$ every 4 weeks for 6 months, with additional doses administered on days 8 and 15 during the first month of therapy only (12).

The study protocol (No. 2793) was approved by the regional Ethical Review Board of the Hyogo College of Medicine.

Surgery. The surgery was performed with the patient in the lateral position. A pneumatic tourniquet was used. A 3-4 cm longitudinally 
Table I. Characteristics and assessment of function, knee instability and sports activity in five male patients who underwent osteoscopic surgery of giant cell tumor of bone.

\begin{tabular}{lccccccccccc}
\hline $\begin{array}{l}\text { Case } \\
\text { no. }\end{array}$ & $\begin{array}{c}\text { Age } \\
\text { (years) }\end{array}$ & Symptom & $\begin{array}{c}\text { Size } \\
(\mathrm{cm})\end{array}$ & $\begin{array}{c}\text { Campanacci } \\
\text { grading }\end{array}$ & $\begin{array}{c}\text { Operative } \\
\text { time }(\mathrm{min})\end{array}$ & $\begin{array}{c}\text { Varus stress } \\
\text { test }\end{array}$ & $\begin{array}{c}\text { Dial } \\
\text { test }\end{array}$ & $\begin{array}{c}\text { Function } \\
(\%)\end{array}$ & $\begin{array}{c}\text { Sport } \\
\text { Tegner } \\
\text { score }\end{array}$ & $\begin{array}{c}\text { Follow-up } \\
(\mathrm{months})\end{array}$ \\
\hline 1 & 59 & Pain & $8.5 \times 4.5 \times 2.5$ & 2 & 120 & $\mathrm{~N}$ & $\mathrm{~N}$ & 100 & Jogging & 5 & 126 \\
2 & 30 & Pain & $6.6 \times 5.0 \times 4.2$ & 2 & 127 & $\mathrm{~N}$ & $\mathrm{~N}$ & 100 & Tennis & 6 & 71 \\
3 & 33 & Pain & $4.5 \times 3.3 \times 2.7$ & 2 & 116 & $\mathrm{~N}$ & $\mathrm{~N}$ & 100 & Tennis & 6 & 40 \\
4 & 17 & Discomfort & $5.6 \times 3.5 \times 3.4$ & 2 & 191 & $\mathrm{~N}$ & $\mathrm{~N}$ & 100 & Soccer & 9 & 30 \\
5 & 57 & Pain & $5.8 \times 5.5 \times 3.8$ & 3 & 157 & $\mathrm{~N}$ & $\mathrm{~N}$ & 100 & Jogging & 5 \\
\hline
\end{tabular}

$\mathrm{N}$, Negative.

curved skin incision was started from $2 \mathrm{~cm}$ proximal of the tip of the proximal fibula. Two round bone fenestrations of $0.5 \mathrm{~cm}$ in diameter were made by high-speed bar as portals (Figure 1) at the anterior and the antero-lataral sides of the fibular head. A $2.7 \mathrm{~mm}$ diameter, $30^{\circ}$ endoscope (Sinuscope; Wolf Stryker Olympus, Hamburg, Germany) was introduced through both portals in order to avoid any blind area of the lesion. Under careful observation of one portal, the tumor was removed through the other portal (Figure 2). Both observation and removal had to be switched between these two portals to avoid any tumor being left behind (2-portal technique) (8). Curettage was achieved using curettes, intervertebral disc Rongeur, shaver blades (Dyonics Power Shaver System; Smith \& Nephew, Andover, MA, USA), and a high-speed bar (High speed drill system; Stryker, Tokyo, Japan) (Figure 3). The bone cavity was irrigated by saline. APC (APC 300; ERBE, Tubingen, Germany) and cementation were used as adjuvants. APC was applied to the entire cavity until all bone surfaces coagulated. We used an argon gas flow of $2 \mathrm{l} / \mathrm{min}$ at a power setting of $40 \mathrm{~W}$ and a maximum irradiation time of $15 \mathrm{~s} / \mathrm{cm}^{2}$ (Figure 4) (13). The coagulum on the bone surface was removed with curettes followed by irrigation with saline. Meanwhile, the bone cavity was observed carefully to find any residual tumor (Figure 5). These procedures, including curettage, APC, and saline irrigation, were repeated at least three times until removal of the entire tumor was confirmed by osteoscopy (Figure 6). Cementation was then performed by filling the bone cavity with polymethylmethacrylate (Surgical Simplex P; Stryker Howmedica Osteonics, Allendale, NJ, USA) (Figure 7).

The patients were allowed to walk the next day after surgery without any aid.

Assessments. Symptoms at presentation were recorded. Size and extension of the tumor were evaluated by magnetic resonance imaging (MRI). Campanacci grading system (14) was used to evaluate cortical breach by knee roentgenograms and computed tomography (CT). Grade 1 lesions have a well-defined margin and an intact or slightly thinned, but not deformed cortex; grade 2 lesions have a relatively well-defined margin but no radiopaque rim, and the cortex is thinned and moderately expanded; grade 3 lesions have blurry borders and cortical destruction with extra-skeletal lesion. CT was also used to assess cortical abnormalities and pathological fractures.

Clinical knee stability was assessed by the varus stress test and the tibial external rotation (dial) test by two physicians independently, and blinded to each other's results. Lateral knee stability was measured by the degree of lateral joint space opening using the varus stress with the knee at 30 degrees of flexion and in neutral tibial rotation. Grade was determined by comparing the results with those for the normal contralateral knee. The amount of lateral opening from 1 to $5 \mathrm{~mm}$ is grade 1 , from 6 to $10 \mathrm{~mm}$ is grade 2 , and greater than $10 \mathrm{~mm}$ is grade 3 . The dial test was used to measure external rotation of the tibia relative to the femur. With the patient in the prone position and the knee flexed at both 30 and 90 degrees, the femur was fixed with one hand while the ankle and foot were externally rotated. An increase of more than 10 degrees of external rotation compared with the contralateral side is a positive test and indicates an injury to the posterior lateral corner (15).

Function was assessed using the rating system of the Musculoskeletal Tumor Society (MSTS) (16), based on six categories: pain, function, emotional acceptance, support, walking ability, and gait. Each category was assigned a numerical value from 0 to 5 points, the higher being better. The values for each category were added, and the functional score is presented as a percentage of maximum values obtainable (30 points). Physical activity was graded by using the Tegner activity scale score (1 to 10) (17). Detailed sports activity was also recorded.

Oncological outcome was assessed by local recurrence and pulmonary metastasis. Local recurrence was evaluated by knee roentgenograms and MRI. CT images were used to detect any pulmonary metastasis. The follow-up of the patients was performed every 3 months over the first 2 years, every 6 months for the next 2 years, and annually thereafter.

\section{Results}

Patient characteristics and results are shown in Table I. Four out of five patients $(80 \%)$ noted pain and one patient (20\%) discomfort. Four patients had a relatively well-defined margin and the cortex was thinned and expanded by the evaluation of the preoperative MRI. These were classified as grade 2 lesions according to the Campanacci grading system (Figure 8). One patient had anterior cortical destruction on preoperative CT images, and the lesion was thus classified as Campanacci grade 3 (case 5 in Table I). After preoperative administration of denosumab (as described above) a cavity was created by a sclerotic wall, with bone healing around the periphery of the tumor (Figure 8). Consequently, the lesion was down-staged from to Campanacci grade 2.

The mean operative time was 142 minutes (range $=116$ to 191 minutes). 
a

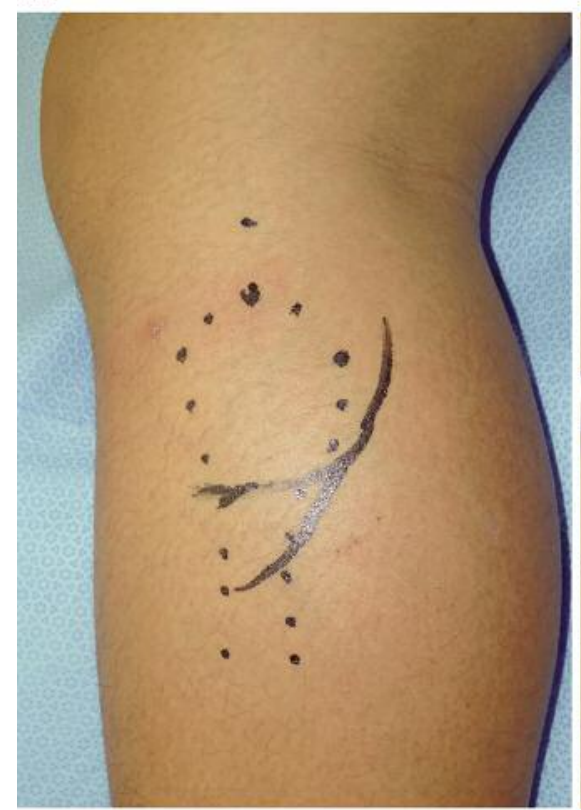

b

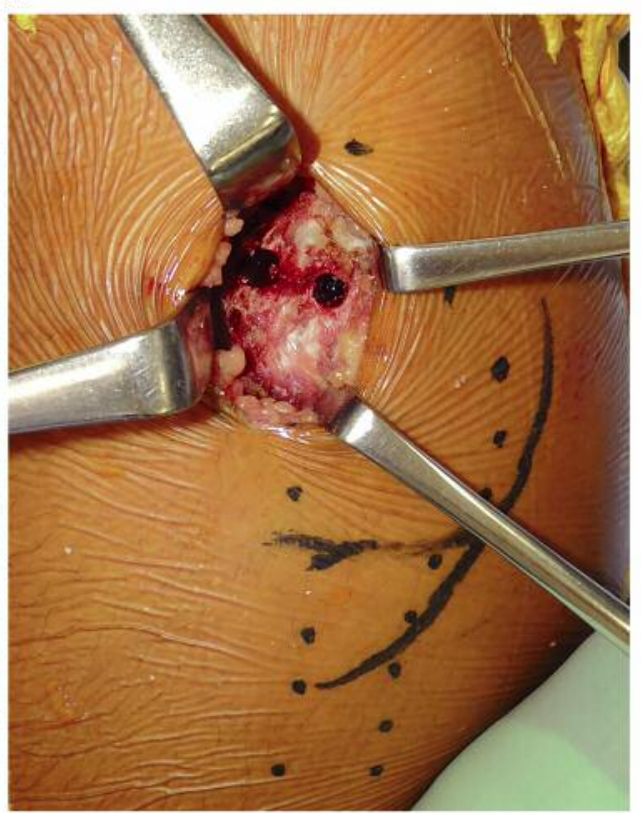

c

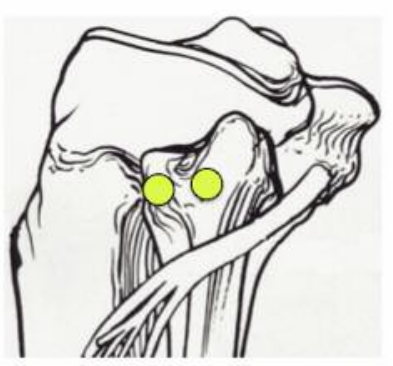

Figure 1. Initial surgical procedure. a: The anatomical locations of the proximal fibula (dotted line) and the common peroneal nerve (solid line) are shown. $b$ : Two round $0.5 \mathrm{~cm}$ in diameter bone fenestrations are made in the anterior and antero-lateral aspect of the fibula head. $c$ : This illustration shows the anatomical location of the two bone fenestrations.

Both the varus stress and the dial tests were negative in all five cases. All of them had 100\% MSTS rating. Tegner activity scores were the same as before surgery. The patients resumed their same sports activities with the same intensity as before.

The mean follow-up was 58 months (range=24-126 months). Neither local recurrence nor pulmonary metastasis has been found among the five cases.

\section{Discussion}

The major clinical importance of the present study is that osteoscopic surgery for GCTB provides immediate functional recovery due to preservation of the proximal fibula. To our knowledge, this is the first report of the novel application of combined osteoscopic curettage and adjuvants (APC and cementation) for the treatment of locally aggressive GCTB. Historically, osteoscopic surgery of bone tumors in extremities was first introduced in 1995 and applied in the case of a chondroblastoma of the femoral head (6). In most of the published cases, osteoscopic surgery was applied for less aggressive benign bone tumors including enchondromas $(7,9)$, bone cysts $(10)$, and intraosseous lipomas (8).

The advantage of osteoscopic curettage over open curettage is the excellent visualization of the bone cavity, which is achieved with a magnified image onto large monitors, even with small cortical fenestrations. Therefore,

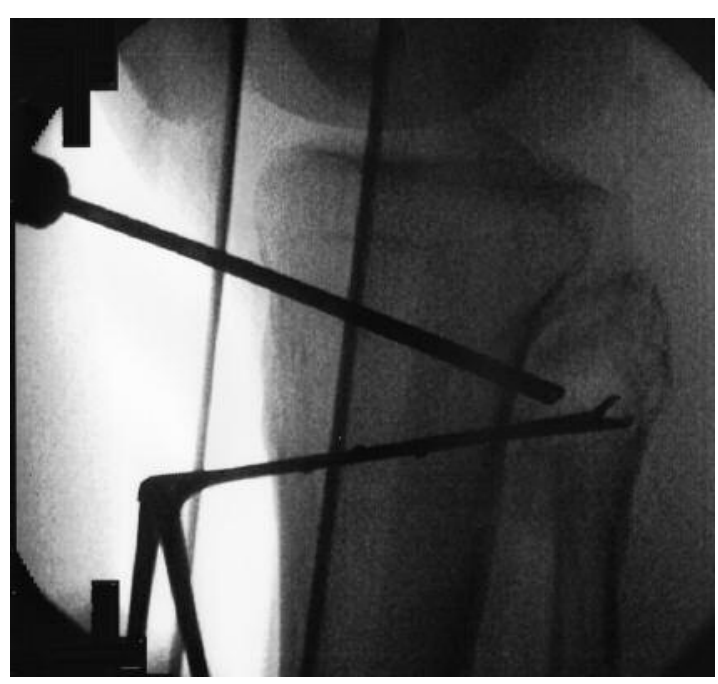

Figure 2. Roentgenogram demonstrating curette and osteoscopy.

complete curettage can be assured since small residual tumors are easily found $(7,8)$. In addition, the use of small cortical fenestrations is far less invasive than that of wide fenestration used in open curettage. Furthermore, by using small cortical fenestrations, the ligament attachments will not be damaged. 


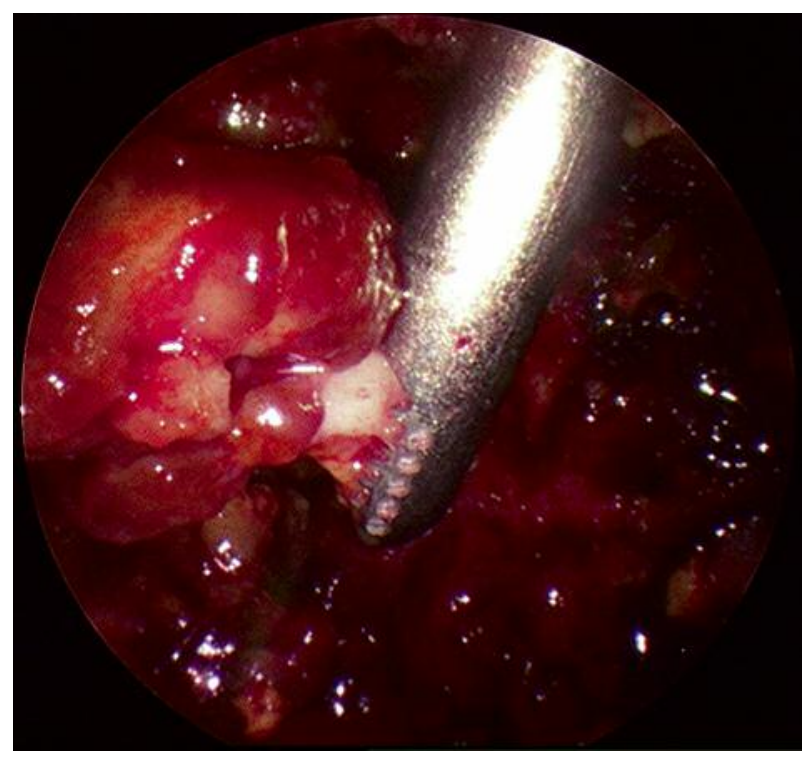

Figure 3. Osteoscopic image showing the curettage of tumor by use of a 3.5-mm shaver blade.

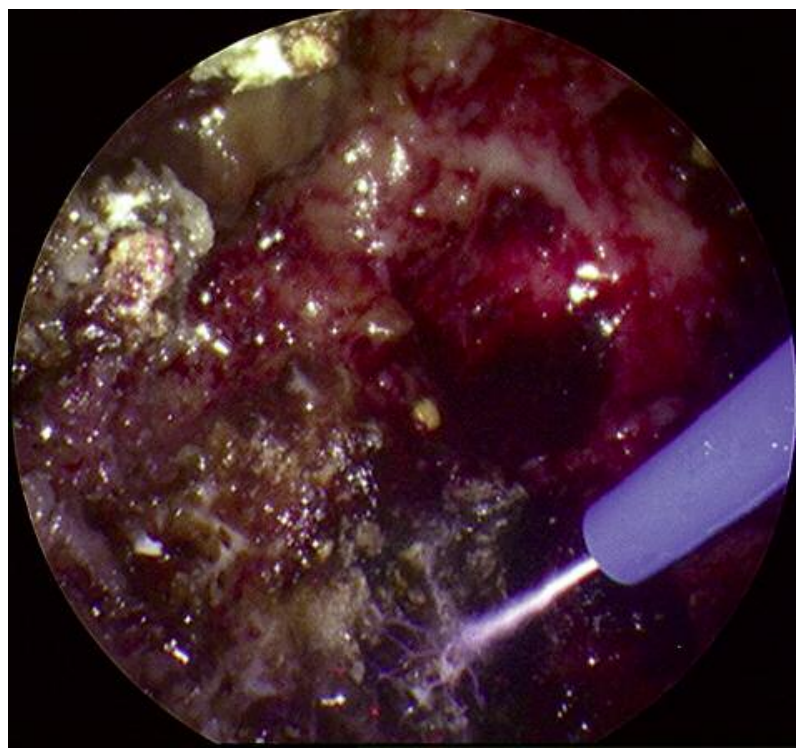

Figure 4. Osteoscopic image showing the argon plasma coagulation after curettage.

The proximal fibula is an anatomically important site for knee stability. Faezypour et al. used en bloc resection with reconstruction in five cases and found grade 1 lateral knee stability in all cases (3). Draganich et al. reviewed six patients in this category, and using the Genucom knee-analysis system, significant differences in knee stability were found in

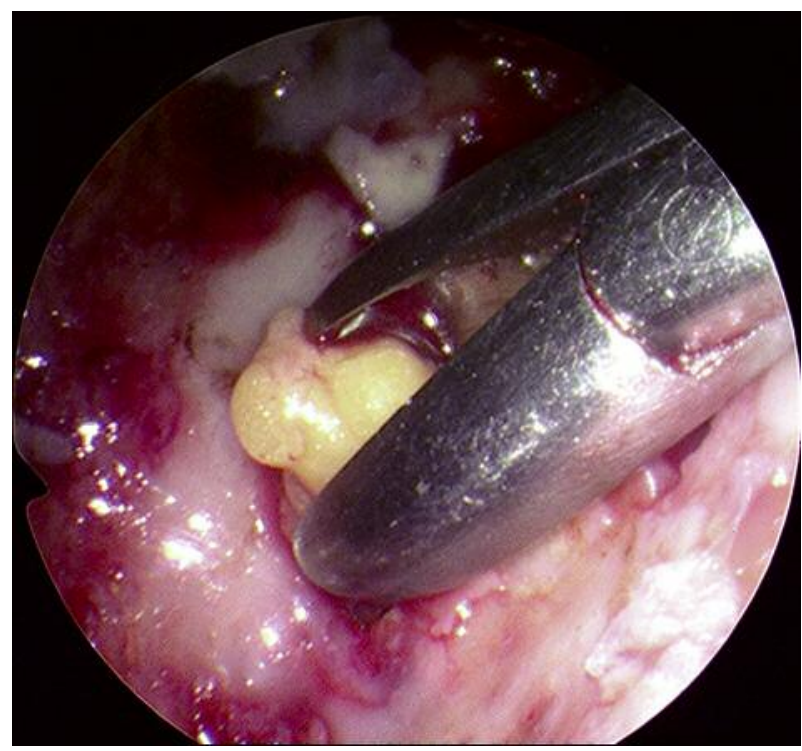

Figure 5. Osteoscopic image showing removal of a residual tumor by use of a curette. The small residual tumor is easily found using magnified images.

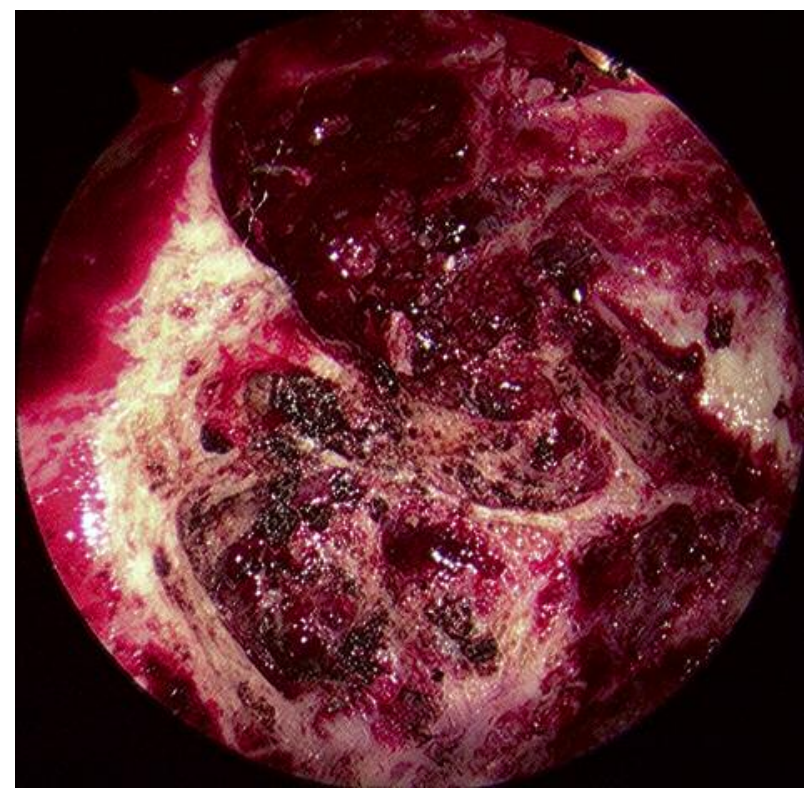

Figure 6. The pitted surface of the inner cortical bone is revealed after curettage and argon plasma coagulation.

comparison to the contralateral side (18). Recently, the posterior lateral corner has been recognized to play important roles for lateral and rotational stability of the knee. The three major static stabilizers of the posterior lateral corner are the LCL, the popliteus tendon and the popliteofibular ligament (PFL). Importantly, two (LCL and PFL) of these attach to the fibular 
a

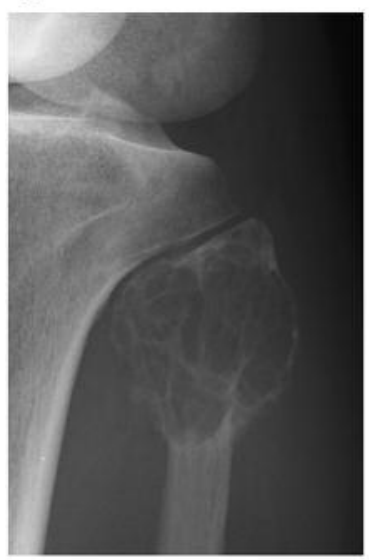

b

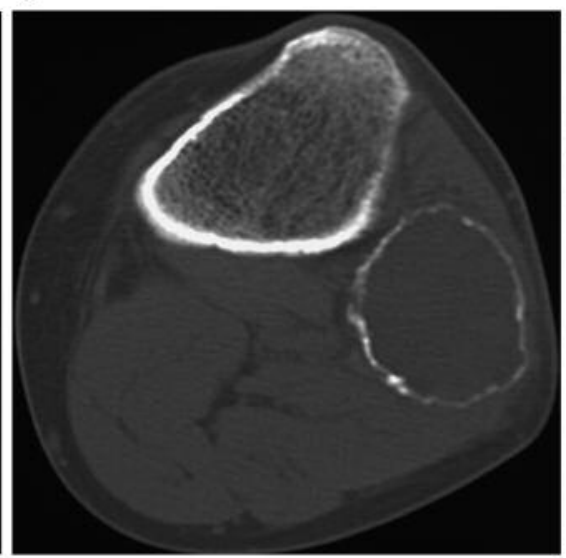

C

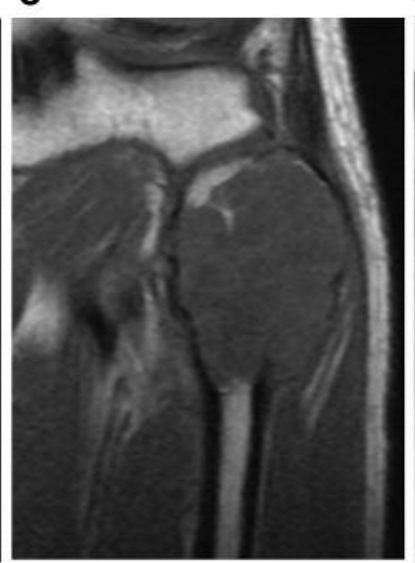

d

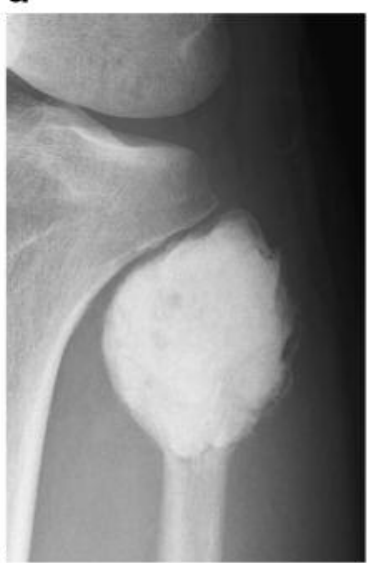

Figure 7. Case 1. a: Preoperative roentgenogram demonstrating the lytic lesion with thinned and expanded cortex. $b$ : Computed tomography (CT) image clearly reveals the lytic lesion with thinned and expanded cortex, which indicates Campanacci grade 2 lesion. $c$ : T1-weighted magnetic resonance image showing the area of decreased signal intensity. $d$ : Postoperative roentgenogram showing well-packed cementation in the bone cavity.

head (19). A cadaveric study revealed that the LCL attaches 8.2 $\mathrm{mm}$ posterior to the anterior aspect of the fibular head. The PFL attaches distally to the posteromedial aspect of the fibular head (20). Therefore, the ideal sites of portals during surgery must be both the anterior and the antero-lateral aspects of the fibula head to avoid damage of the posterior lateral corner. In the present study, there was neither lateral nor external rotational instability, as shown by negative results of the varus stress and dial tests.

Furthermore, use of the anterior aspect of the fibula head for portal access has the advantage of avoidance of the risk of peroneal nerve palsy due to its anatomically safe distance from the common peroneal nerve. No peroneal nerve palsy was found in the present study. In contrast, after en bloc resection of the proximal fibula, the rates of peroneal nerve palsy reportedly range from 20 to $67 \%(2,5,21)$. Due to the anatomical proximity of the common peroneal nerve, an expanded GCTB may elevate and stretch the nerve, which again may result in spontaneous or iatrogenic neurological complications related to surgical interventions $(3,4)$.

With regard to functional outcome, the present study showed excellent results using osteoscopic surgery. Zhao et al. reported that mean MSTS ratings were $98 \%$ in cases with reconstruction (seven out of 11 cases with GCTB in the proximal fibula), and $72 \%$ in cases without reconstruction (four out of 11 cases) (22). In a different case series, Faezypour et al. reported that five patients with GCTB in the proximal fibula could not return to high-demand sports even though reconstruction was performed (3). In the present study, all patients were able to walk and even returned to sports activities from the next day after surgery without any rehabilitation.

A challenging issue regarding management of GCTB is the high local recurrence rate, in particular after open curettage alone. Abdel et al. showed that the local recurrence rate could a

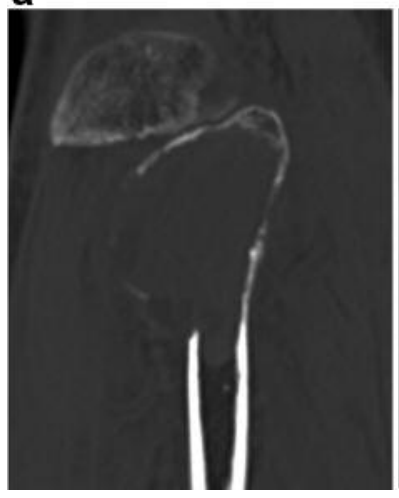

b

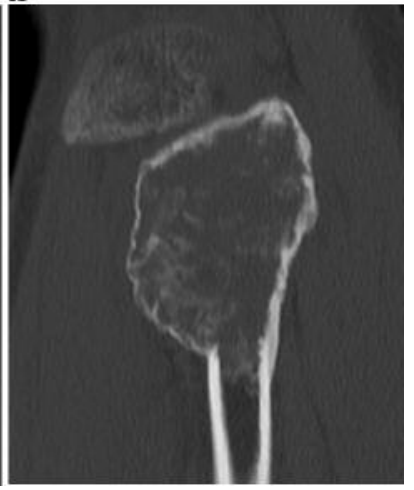

Figure 8. Case 5. a: Sagittal multiplanar reformation image of computed tomography showing anterior cortical destruction, consistent with Campanacci grade 3 lesion. $b$ : After denosumab administration, a sclerotic wall can be seen to have formed along the anterior aspects of the peripheral tumor regions.

be reduced by en bloc resection (two out of 18 cases, 11\%) in comparison to open curettage without adjuvant (two out of three cases, 67\%) (4). Their study has the highest number of cases $(n=21)$ reported with GCTB in the proximal fibula.

The combination of APC and cementation has been reported as intensely effective. In a series of 37 patients with GCTB (several bone locations) treated with open curettage and these adjuvants, only four (11\%) had local recurrence (23), which is the same rate as en bloc resection of the proximal fibula reported by Abdel et al. (4). Advantages of APC are precise local control of the device and no risk of chemical toxicity (22). In addition, APC is characterized by 
non-contact coagulation, making it possible to coagulate a wide range target uniformly (13). In fact, no local recurrence was found in any of our five cases, indicating that sufficient tumor resection was obtained by osteoscopic surgery.

The limitation of osteoscopic surgery is that it cannot be applied in cases with extra-skeletal lesions. However, in one such case in the present study (case 5), denosumab was used to effectively create a sclerotic wall, and subsequently osteoscopic surgery was possible. Cases are not suitable for this technique if there is no effect of denosumab.

The major limitation of this study was the small number of patients; most previous reports on GCTB in the proximal fibula from single institutes comprised fewer than five cases due to the rarity of this condition. A multicenter study should be conducted in the future.

In conclusion, the technique of osteoscopic surgery is feasible for patients with GCTB in the proximal fibula in order to preserve this anatomically important site, and thereby obtain immediate functional recovery and avoid a long-term period of immobilization. This technique can improve the quality of life for patients with GCTB in the proximal fibula, with satisfactory oncological outcome.

\section{Conflicts of Interest}

Authors have no conflicts of interest in regard to this study.

\section{Acknowledgements}

This work was in part supported by a Grant-in-Aid (C) (22591671) from the Ministry of Education, Culture, Sports, and Science.

\section{References}

1 Unni KK, Inwards CY (eds): Dahlin's Bone Tumors: General Aspects and Data on 10,165 Cases. 6th. Lippincott; Williams \& Wilkins, Philadelphia, PA. Giant cell tumor (osteoclastoma), pp. 225-242, 2010.

2 Gitelis S, Mallin BA, Piasecki P and Turner F: Intralesional excision compared with en bloc resection for giant-cell tumors of bone. J Bone Joint Surg Am 75: 1648-1655, 1993.

3 Faezypour H, Davis AM, Griffin AM and Bell RS: Giant cell tumor of the proximal fibula: surgical management. J Surg Oncol 61: 34-37, 1996.

4 Abdel MP, Papagelopoulos PJ, Morrey ME, Wenger DE, Rose PS and Sim FH: Surgical management of 121 benign proximal fibula tumors. Clin Orthop Relat Res 468: 3056-3062, 2010.

5 Malawer MM: Surgical management of aggressive and malignant tumors of the proximal fibula. Clin Orthop Relat Res 186: 172$181,1984$.

6 Thompson MS and Woodward JS Jr.: The use of the arthroscope as an adjunct in the resection of a chondroblastoma of the femoral head. Arthroscopy 11: 106-111, 1995.

7 Sekiya I, Matsui N, Otsuka T, Koayashi M and Tsuchiya D: The treatment of enchondromas of the hand by endoscopic curettage without bone grafting. J Hand Surg Br 22: 230-234, 1997.
8 Futani H, Fukunaga S, Nishio S, Yagi $M$ and Yoshiya S: Successful treatment of bilateral calcaneal intraosseous lipomas using endoscopically assisted tumor resection. Anticancer Res 27: 4311-4314, 2007.

9 Taleb C, Gouzou S, Mantovani G and Liverneaux P: Treatment of benign bone tumours of the hand using osteoscopy. Chir Main 29: 78-81, 2010.

10 Nishimura A, Matsumine A, Kato K, Aasanuma K, Nakamura T, Fukuda A and Sudo A: Endoscopic versus open surgery for calcaneal bone cysts: A preliminary report. J Foot Ankle Surg 55: 782-787, 2016.

11 Thomas DM, Thomas D, Henshaw R, Skubitz K, Chawla S, Staddon A, Blay JY, Roudier M, Smith J, Ye Z, Sohn W, Dansey R and Jun S: Denosumab in patients with giant-cell tumour of bone: an open-label, phase 2 study. Lancet Oncol 11: 275-280, 2010.

12 Rutkowski P, Ferrari S, Grimer RJ, Stalley PD, Dijkstra SP, Pienkowski A, Vaz G, Wunder JS, Seeger LL, Feng A, Roberts ZJ and Bach BA: Surgical downstaging in an open-label phase II trial of denosumab in patients with giant cell tumor of bone. Ann Surg Oncol 22: 2860-2868, 2015.

13 Sagawa T, Takayama T, Oku T, Hayashi T, Ota H, Okamoto T, Muramatsu H, Katsuki S, Sato Y, Kato J and Niitsu Y: Argon plasma coagulation for successful treatment of early gastric cancer with intramucosal invasion. Gut 52: 334-339, 2003.

14 Campanacci M, Baldini N, Boriani S and Sudanese A: Giant-cell tumor of bone. J Bone Joint Surg Am 69: 106-114, 1987.

15 Covey DC: Injuries of the posterolateral corner of the knee. J Bone Joint Surg Am 83-A: 106-118, 2001.

16 Enneking WF, Dunham W, Gebhardt MC, Malawar M and Pritchard DJ: A system for the functional evaluation of reconstructive procedures after surgical treatment of tumors of the musculoskeletal system. Clin Orthop Relat Res 286: 241-246, 1993.

17 Tegner Y and Lysholm J: Rating systems in the evaluation of knee ligament injuries. Clin Orthop Relat Res 198: 43-49, 1985.

18 Draganich LF, Nicholas RW, Shuster JK, Sathy MR, Chang AF and Simon MA: The effects of resection of the proximal part of the fibula on stability of the knee and on gait. J Bone Joint Surg Am 73: 575-583, 1991.

19 Ranawat A, Baker CL 3rd, Henry $S$ and Harner CD: Posterolateral corner injury of the knee: evaluation and management. J Am Acad Orthop Surg 16: 506-518, 2008.

20 LaPrade RF, Ly TV, Wentorf FA and Engebretsen L: The posterolateral attachments of the knee: a qualitative and quantitative morphologic analysis of the fibular collateral ligament, popliteus tendon, popliteofibular ligament, and lateral gastrocnemius tendon. Am J Sports Med 31: 854-860, 2003.

21 Erler K, Demiralp B, Ozdemir MT and Basbozkurt M: Treatment of proximal fibular tumors with en bloc resection. Knee 11: 489496, 2004.

22 Zhao SC, Zhang CQ and Zhang CL: Reconstruction of lateral knee joint stability following resection of proximal fibula tumors. Exp Ther Med 7: 405-410, 2014.

23 Lewis VO, Wei A, Mendoza T, Primus F, Peabody T and Simon MA: Argon beam coagulation as an adjuvant for local control of giant cell tumor. Clin Orthop Relat Res 454: 192-197, 2007.

Received January 28, 2018

Revised March 6, 2018

Accepted March 8, 2018 